\title{
Treatment of hypertension during pregnancy
}

David Hall

Department of Obstetrics and Gynaecology, University of

Stellenbosch and Tygerberg Academic Hospital, South Africa

Address for correspondence:

Prof DR Hall

Department of Obstetrics and Gynaecology

Tygerberg Hospital and University of Stellenbosch

PO Box 19081

Tygerberg

7505

South Africa

Email:

drh@sun.ac.za

\section{INTRODUCTION AND CONTEXT}

Hypertension is the most common medical complication encountered during pregnancy, complicating 5-15\% of all pregnancies. ${ }^{(1,2)}$ In a systematic review, Abalos, et al. reported upon global and regional estimates of pre-eclampsia and eclampsia from 40 countries. Their model gave an estimated incidence of $4.6 \%$ and $1.4 \%$ for pre-eclampsia and eclampsia respectively, but they noted wide variations across regions. ${ }^{(3)}$ With the increasing prevalence of obesity and diabetes as well as the trend to more advanced age at childbirth in many countries, the rates of pre-existing (chronic) and pregnancyinduced hypertensive conditions such as pre-eclampsia seem set to rise even further. ${ }^{(4)}$

The impact of hypertensive diseases on pregnancy is certainly important from both maternal and fetal perspectives during pregnancy and even thereafter. The maternal mortality associated with these conditions remains high in both developed ${ }^{(5)}$ and developing countries. ${ }^{(6,7)}$ In South Africa, a legislated confidential enquiry process has facilitated the expert assessment of all maternal deaths since 1999. The category of "hypertensive disorders in pregnancy" has consistently remained an important primary cause of death. During the 2008-2010 triennium, hypertensive disorders during pregnancy accounted for $14 \%$ of all maternal deaths in South Africa. This figure represented a continued decrease from 19\% in 2002-2004 and 16\% in 2005-2007. In the most recent triennium there were 679 deaths reported in the hypertension category. Of these, 5\% were due to chronic hypertension and the rest due to preeclampsia including superimposed and complicated pre-

\section{ABSTRACT}

Hypertensive disorders of pregnancy are on the increase and remain important causes of maternal and perinatal morbidity and mortality. The different classes of hypertension carry different risks but severe hypertension in itself remains a medical emergency. Pre-eclampsia is a particularly dangerous condition that may progress rapidly. During pregnancy mild hypertension without end-organ disease does not require treatment until levels of $150 / 100 \mathrm{mmHg}$ are reached. Acute severe hypertension must be diagnosed and treated expeditiously. The choice of anti-hypertensive agents is limited in pregnancy and physicians should choose agents which are regarded as safe and which they feel comfortable in using. After acute management, oral maintenance therapy will be indicated in many cases, although delivery is regarded as the only definitive treatment for pregnancy specific conditions such as pre-eclampsia. Previous pre-eclampsia is associated with increased rates of cardiovascular and metabolic disease in later life. This should prompt subsequent lifestyle education and intervention. SAHeart 20 | 4; 1 1:68-74

eclampsia. Amongst these women, pulmonary oedema (28\%), intracranial haemorrhage (23\%), cerebral oedema resulting in coning (9\%) and brain death following a hypoxic event (7\%) were the most frequently recorded final causes of death. Although most of these deaths occurred in tertiary and regional hospitals, hypertensive deaths still comprised 15\% of all maternal deaths at Community Health Centres, emphasising the importance of correct initial management at all levels of care. ${ }^{(6)}$ With regards to the timing of the emergency event related to hypertension, $28 \%(n=|9|)$ occurred in the postpartum period. An important aspect of the Saving Mothers report is the attention paid by assessors to avoidable factors, missed opportunities and sub-standard care. In the most recent report, health worker orientated emergency management problems were noted in $42 \%$ of hypertensive deaths at Community Health Centres and District hospitals, 33\% at Regional hospitals and 17\% at Tertiary hospitals. ${ }^{(6)}$ Not only is the mortality associated with hypertensive disorders in pregnancy high, but these conditions are a frequent source of significant morbidity. ${ }^{(8,9)}$ Cases with Severe Acute Maternal Morbidity (SAMM) share many characteristics with maternal deaths. ${ }^{(10)}$ Therefore understanding and managing SAMM also helps to decrease or prevent maternal mortality. 
From the fetal and neonatal perspective, hypertensive diseases of pregnancy remain important causes for delivery of very low birthweight babies, ${ }^{(1)}$ preterm births (with the risk of intracerebral haemorrhage) and perinatally related losses, that are often caused by placental abruption and/or intra-uterine growth restriction. ${ }^{(12-14)}$ The perinatal outcomes of the 679 hypertensive maternal deaths that occurred in the 2008-2010 triennium in South Africa included 144(21\%) stillbirths and 18(2.7\%) neonatal deaths, while $226(33.3 \%)$ of the women died undelivered. ${ }^{(6)}$

Anti-hypertensive therapy is used in the acute and non-acute clinical scenario. With regards to the non-acute management of the hypertensive diseases of pregnancy, Rubin, in 1986 , noted a shift away from non-pharmacological management regimens, such as bed rest or early delivery, towards the introduction of anti-hypertensive agents with a view to useful prolongation of pregnancy. ${ }^{(15)}$ Clinicians using anti-hypertensive agents during pregnancy have to balance both the benefits and the risks of such therapy while considering maternal and fetal interests. Although a wide variety of agents have been used, few have been subject to controlled clinical trials, due to concerns harboured by health research ethics committees and pharmaceutical companies. ${ }^{(16)}$ The result is that many medications are prescribed off-label in doses and frequencies extrapolated from research in non-pregnant subjects with a very different physiology. This problem is particularly applicable to anti-hypertensive agents and remains an ethical conundrum.

\section{PHYSIOLOGY}

Several important physiological changes occur during normal and even complicated pregnancies. It is well known that blood pressure is directly proportional to systemic vascular resistance and cardiac output. Starting early in the first trimester and peaking around the 28th week of gestation, the plasma and red cell volumes increase by $40 \%$ and $25 \%$ respectively. The greater increment in plasma volume gives rise to the physiologic anaemia of pregnancy. Cardiac output increases by $40 \%$ during pregnancy and a further $20 \%$ during labour. Apart from the plasma volume expansion, there is vasodilatation leading to a fall in blood pressure during the second trimester of pregnancy. This is due to several factors that include vascular refractoriness to angiotensin II, as well as increased production of endothelial prostacyclin and nitric oxide.(17) The nadir in systolic and diastolic pressure is reached at about 22-24 weeks' gestation. Thereafter there is a steady rise to prepregnant levels at term. Immediately after delivery, blood pressure usually falls and then rises again up to the third or fourth day postpartum. The physiological lowering of blood pressure in the second trimester may obscure the diagnosis of mild chronic hypertension in women without the benefit of pre-pregnancy check-ups and who only book for antenatal care in the second trimester. When blood pressure remains elevated beyond 12 weeks after delivery, the diagnosis of chronic hypertension is confirmed. ${ }^{(18)}$

\section{CLASSIFICATION}

There are several classification frameworks for hypertension in pregnancy, some being more complex than others. Steegers, et al. have summarised the main differences between classifications as: inclusion of non-proteinuric pre-eclampsia; differentiation between research and clinical definitions; the acceptance of early pre-eclampsia as severe; the definition of severe pre-eclampsia and the importance of white-coat hypertension. ${ }^{(19)}$ The classification of hypertension in pregnancy of the International Society for the Study of Hypertension in Pregnancy (ISSHP) is in the final stages of review but key aspects of their current classification are shown in Table I.

While most classifications use fixed thresholds it is still recommended that a pregnant woman with a rise in $\mathrm{sBP} \geq 30 \mathrm{mmHg}$ or $\mathrm{dBP} \geq 15 \mathrm{mmHg}$ should be carefully monitored. ${ }^{(17)}$ As seen in Table I, hypertension in pregnancy can be broadly divided into conditions of pre-existing hypertension, pregnancy-induced hypertension (gestational hypertension) and pre-eclampsia that manifest clinically after 20 weeks' gestation. Some women will not have been diagnosed with chronic hypertension but

\section{TABLE I: Key aspects from the classification and diagnosis of the hypertensive disorders of pregnancy by the International Society for the Study of Hypertension in Pregnancy (ISSHP). ${ }^{(20)}$}

Definition of hypertension in pregnancy

A systolic blood pressure (sBP) $\geq 140 \mathrm{mmHg}$ and or a diastolic (dBP) $\geq 90 \mathrm{mmHg}(\mathrm{K} 5)$ respectively.

\section{Classification}

Four categories: pre-eclampsia, gestational hypertension, chronic hypertension (essential and secondary), and pre-eclampsia superimposed on chronic hypertension. The definitions are listed:

Pre-eclampsia: The clinical diagnosis is as follows:

- De novo hypertension after gestational week 20 , and new onset of one or more of the following

- Proteinuria ( $>300 \mathrm{mg} /$ day or a spot urine protein/creatinine ratio $>30 \mathrm{mg} / \mathrm{mmol}$ )

- Renal insufficiency (creatinine $>90 \mu \mathrm{mol} / \mathrm{L}$ or oliguria)

- Liver disease (raised transaminases and/or severe right upper quadrant or epigastric pain)

- Neurological problems: convulsions (eclampsia), hyperreflexia with clonus, severe headaches with hyperreflexia, persistent visual disturbances (scotoma)

- Haematological disturbances: thrombocytopenia, disseminated intravascular coagulation, haemolysis

- Fetal growth restriction.

Normalisation of blood pressure within 3 months postpartum.

Gestational hypertension: De novo hypertension alone, appearing after gestational week 20 .

Chronic hypertension: Presence or history of hypertension preconception or in the first half of pregnancy. Considered "essential" if there is no underlying cause or "secondary" if associated with definitive etiology.

Pre-eclampsia superimposed on chronic hypertension: Development of new signs and/or symptoms associated with pre-eclampsia after gestational week 20, as above, in a woman with chronic hypertension. 
if hypertension is noted before 20 weeks' gestation, it is likely to be a pre-existing problem. In such cases secondary causes e.g. renal, cardiac and endocrine should be excluded before a diagnosis of essential hypertension is made. Women with chronic hypertension are at increased risk of developing superimposed pre-eclampsia. Pre-eclampsia is a pregnancy specific and particularly dangerous condition with multifactorial etiology that is generally characterised by new onset hypertension and proteinuria after 20 weeks' gestation. Pre-eclampsia may be complicated by eclampsia (grand mal convulsions), blindness, pulmonary oedema, haemolysis, elevated liver enzymes and low platelets (HELLP) syndrome, renal failure, placental abruption, intra-uterine growth restriction (IUGR) or intra-uterine death (IUD) of the fetus. A detailed discussion of pre-eclampsia is however beyond the scope of this article. Apart from the problems of pre-eclampsia, any form of severe hypertension is dangerous due to the association with stroke, end-organ damage and placental abruption. ${ }^{(17,21)}$

\section{DIAGNOSING HYPERTENSION AND TREATMENT GOALS}

The Millennium Development Goal number 5 requires a specific reduction of maternal mortality by 2015 . When one considers the number of hypertensive deaths occurring annually in South Africa, it is clear that the identification of hypertension during pregnancy as well as treatment towards the prevention and management of hypertensive crises will help towards achieving this laudable goal. The implementation of clinical guidelines for treatment and referral of pregnant women with hypertension has been shown to reduce maternal and perinatal risk. ${ }^{(22)}$

General antenatal care (history, checking blood pressure and urine) is largely directed toward the screening and detection of hypertension and pre-eclampsia which may be asymptomatic in the early stages. Blood pressure should be measured as it is done outside of pregnancy with the patient at rest, sitting upright, arms supported and the cuff at the level of her heart. When measured in the recumbent position, the patient should lie on her side, again with the cuff at the level of her heart. Korotkoff $\vee$ sounds should be used to define diastolic blood pressure. Obesity is a problem and "large" cuffs should be used when the circumference of the upper arm is $\geq 33 \mathrm{~cm}$. Another matter to consider is the device used to measure blood pressure. The mercury sphygmomanometer has been the gold standard but has been removed from many hospitals and clinics due to safety concerns. The replacement devices are generally aneroid and automated machines, but only a few of the automated blood pressure measuring devices on the market have been validated for use in pregnancy. The lower threshold for diagnosing hypertension is generally agreed upon (see Table I) but the definition of severe systolic hypertension $(160 \mathrm{mmHg}$ or $170 \mathrm{mmHg}$ ) is still debated due to concerns about lethal maternal stroke. ${ }^{(19,21)}$ At Tygerberg Academic Hospital, severe hypertension in pregnancy is defined as systolic blood pressure $(\mathrm{sBP}) \geq 160 \mathrm{mmHg}$ or diastolic blood pressure $(\mathrm{dBP}) \geq 110 \mathrm{mmHg}$. Non-severe hypertension is defined as sBP $140-159 \mathrm{mmHg}$ and dBP $90-109 \mathrm{mmHg}$. Parenteral antihypertensive therapy may be necessary to control acute severe hypertension but this can be followed by oral maintenance therapy.

There is currently no universally accepted standard of care although there is broad agreement on the principles of assessment, surveillance, management of severe systolic and diastolic blood pressures, prevention and treatment of eclampsia and avoidance of aggressive rehydration in preeclampsia. In contrast to the non-pregnant scenario, therapy for moderate hypertension in pregnancy or pre-eclampsia is controversial. This is because women with mild, uncomplicated hypertension without end-organ involvement are at lower risk of adverse outcomes over the "short period" that pregnancy represents. ${ }^{(23)}$ Due to the mid-trimester fall in blood pressure, it may be appropriate to stop anti-hypertensive medication in uncomplicated pre-existing hypertension, only re-instating it if required. Many physicians will still treat non-severe hypertension in pregnancy in order to reduce the risk of severe hypertension and maternal end-organ damage when the blood pressure rises above $150 / 100 \mathrm{mmHg}$, but this has not been shown to decrease super-imposed pre-eclampsia. ${ }^{(1,2,24)}$ Systolic blood pressure is now regarded as the strongest predictor of maternal end-organ damage. ${ }^{(21)}$ With prolonged treatment of non-severe hypertension, fetal growth restriction due to placental hypoperfusion remains a concern, hence the importance of fetal surveillance. A Cochrane review of 46 trials, including 4282 women, concluded that it remains unclear whether antihypertensive drug therapy for mild/moderate hypertension in pregnancy is worthwhile. . $^{(25)}$

While therapy for moderate hypertension or pre-eclampsia is controversial, it is well accepted in the severe form as severe hypertension is associated with more adverse outcomes. ${ }^{(18)}$ However, because the placenta functions as a low resistance shunt without autoregulation, lowering the blood pressure too rapidly or too far is detrimental to the fetus. In the acute phase, the aim is an initial reduction of $25 \%$ in the first hour, followed by further reduction over hours to values below $160 / 110 \mathrm{mmHg}$. ${ }^{(18)}$ Thereafter, the target range is $140-155 \mathrm{sBP} /$ 90-105dBP. A summary of the treatment thresholds and targets of 5 societies is provided by Vest and Cho.(1) In severe hypertension or severe pre-eclampsia the primary purpose of therapy is not to change the course of the illness but to prevent cerebral haemorrhage and hypertensive encephalopathy. Thus the initial focus for the benefit of therapy is directed towards the safety of the mother. It is now known that the fetus too can benefit from such therapy when it allows the careful continuation of pregnancy remote from term. Regarding severe pre-eclampsia, 2 randomised controlled trials have shown that expectant management of early onset, severe pre-eclampsia is of benefit to premature fetuses. ${ }^{(26,27)}$ These and other studies 
have shown that perinatal outcome can be directly linked to gestation at delivery. ${ }^{(28)}$ Very preterm delivery is associated with the significant risks of pulmonary immaturity and the development of neurocognitive compromise. The administration of betamethasone to enhance fetal pulmonary maturity and decrease fetal intraventricular haemorrhage and necrotising enterocolitis, together with a short but significant prolongation of pregnancy, can greatly improve the neonatal outcome. Due to the fact that approximately 2 weeks of gestation can be gained by expectant management, oral rather than parenteral therapy is the appropriate choice for this longer period of therapy. When anti-hypertensive medications are used to control blood pressure and postpone delivery in pre-eclampsia with the goal of improving fetal outcome, this is only a palliative step and the disease process itself is not modified. The timing of delivery and balancing of the maternal and fetal risks remains a critical decision.

The care of pregnant women with hypertension does not end with delivery. Hypertension caused by pre-eclampsia usually resolves within a few days of delivery and returns to baseline by 12 weeks postpartum.(1) During the post-partum period many women will require ongoing control of hypertension with oral agents. Labetalol, nifedipine, enalapril and captopril are safe during lactation but other ACE inhibitors and angiotensin receptor blockers should not be prescribed. ${ }^{(29)}$ For women who entered pregnancy with normal blood pressure it is generally recommended to discontinue antihypertensive medications 3-4 weeks after delivery and then to observe blood pressure at regular intervals for one month and then yearly thereafter. ${ }^{(1)}$

Recently, compelling evidence associating hypertensive disorders in pregnancy with cardiovascular risk factors, has been presented. ${ }^{(30)}$ A very large study from Norway ${ }^{(31)}$ together with others from around the world offer plausible evidence that women who experienced pregnancy-related hypertension are at elevated risk for hypertension and metabolic disorders such as non-insulin dependent diabetes mellitus later in life. . $^{(30,32)}$ Although it is still uncertain whether the associations between pregnancy hypertension and subsequent chronic disease are due to common pre-existing conditions or by the direct effect of the complication during pregnancy, the former hypothesis is currently thought to be more likely. ${ }^{(4)}$ This debate should however not delay the implementation of simple measures aimed at improving long-term outcomes such as smoking cessation, diet, management of obesity, exercise programmes and regular checking of blood pressure, lipid and glucose profiles. Cardiologists in particular should take note of these associations.

\section{ANTI-HYPERTENSIVE AGENTS}

The management of pregnant women with hypertension is based on the following principles:

Exclude secondary causes of hypertension when hypertension presents before 20 weeks' gestation.
Exclude or confirm pre-eclampsia after 20 weeks' gestation.

Treat the hypertension.

Fetal surveillance.

Decide on timing and type of delivery.

In this section only the third principle will receive detailed attention but readers are encouraged to read more widely, particularly regarding pre-eclampsia. Regardless of the underlying pathology, severe hypertension merits treatment in its own right, because when the mean arterial pressure rises above 150 there is loss of cerebral auto-regulation and a risk of cerebral haemorrhage. However, in pre-eclampsia cerebral complications can occur without very high blood pressure as blood pressure does not always correlate with auto-regulation functionality. ${ }^{(33)}$ As stated in the introduction there are insufficient randomised trials to direct the optimal treatment of hypertension in pregnancy, thus physicians should use the agent with which they feel most comfortable. ${ }^{(34)}$

\section{Hypertensive emergencies}

Therapy for acute severe hypertension is essential. The primary purpose of therapy is not to change the course of the illness but to prevent cerebral haemorrhage, hypertensive encephalopathy, acute renal or cardiac failure, arrhythmias or placental abruption. Although the initial focus on the benefit of therapy is directed towards the safety of the mother whose well-being takes priority in the acute phase, the fetus too benefits from such therapy, particularly when it allows the careful continuation of pregnancy remote from term. If the reduction in blood pressure is too rapid, this may lead to inadequate uteroplacental blood flow. A fast but smooth and controlled reduction is the ideal. Careful fetal monitoring to detect fetal distress is required but maternal well-being takes priority in the acute phase. Women with acute severe hypertension should be stabilised and transferred to, or managed in a high-care dependency unit. Physicians should be aware that automated oscillometric devices may underestimate blood pressure. ${ }^{(35)}$ Women with pre-eclampsia and acute severe hypertension have a reduced intravascular volume and require pre-treatment with a small volume of fluid e.g. $250 \mathrm{ml}$ crystalloid fluid before or during administration of anti-hypertensive medication. ${ }^{(36)}$ The following agents are commonly used. Drug safety classes are shown in Table 2.

Nifedipine (Class C) $5 \mathrm{mg}$ or $10 \mathrm{mg}$ (short-acting capsule) swallowed. Note: In the pregnant patient the capsule should not be bitten or administered sub-lingually. A rapid effect on the blood pressure can still be anticipated. It may be repeated every 30 minutes as needed. Nifedipine should be avoided in women with known atherosclerotic cardiovascular disease, a long history of diabetes ( $>15$ years) or $>45 y e a r s$ of age due to the risk of sudden cardiac death. ${ }^{(37)}$ 
- Dihydralazine (Class C) Mix one ampoule with $20 \mathrm{ml}$ sterile water. Administer $2 \mathrm{ml}(2.5 \mathrm{mg})$ boluses intravenously every 20 minutes until blood pressure is $<160 / 110 \mathrm{mmHg}$. When the patient is ready for transfer, administer $6.25 \mathrm{mg}$ intramuscularly. It has been associated with more side-effects than other rapid-acting agents. ${ }^{(38,39)}$

- Labetalol (Class C) When maternal tachycardia accompanies acute severe hypertension labetalol is a good option. A $20 \mathrm{mg}$ intravenous bolus followed by $40 \mathrm{mg}$ if not effective within 10 minutes; then $80 \mathrm{mg}$ every 30 minutes to a maximum total dose of $220 \mathrm{mg}$. It may also be used as a continuous infusion.

Magnesium sulphate $\left(\mathrm{MgSO}_{4}\right)$ (Class B) This drug is not an anti-hypertensive agent but is used for the treatment and prevention of eclampsia in the acute scenario. A $4 \mathrm{~g}$ intravenous loading dose over 15 minutes is followed by an infusion of Ig/hour.

The aim here is not to normalise the blood pressure, but rather to reduce the pressure to levels associated with a decreased risk of vascular accidents. In the acute scenario, the aim is to reduce the $\mathrm{sBP} / \mathrm{dBP}$ to $<160 / 110 \mathrm{mmHg}$ while recording the blood pressure every five minutes until stable. In a meta-analysis investigating treatment for severe hypertension in pregnancy, Magee, et al. concluded that hydralazine lacked support as a first line agent and that nifedipine and labetalol showed promise. ${ }^{(38)}$ In 2013 a small randomised controlled trial comparing oral nifedipine to intravenous labetalol for hypertensive emergencies in pregnancy showed nifedipine to be superior. ${ }^{(40)}$ Following control of severe hypertension, oral maintenance therapy should be instituted or increased.

TABLE 2: Drug rating in pregnancy. ${ }^{(17)}$

A Controlled studies show no risk Adequate, well-controlled studies in pregnant women have failed to demonstrate risk to the fetus.

B No evidence of risk in humans Either animal findings show risk (but human findings do not) or, if no adequate human studies have been done, animal findings are negative.

C Risk cannot be ruled out Human studies are lacking and animal studies are either positive for fetal risk or lacking as well. However, potential benefits may justify the potential risk.

D Positive evidence of risk Investigational or post marketing data show risk to fetus. Nevertheless, potential benefits may outweigh the risk.

$X \quad$ Contra-indicated in pregnancy

Studies in animals or humans, or investigational or post marketing reports have shown fetal risk which clearly outweighs any possible benefit to the patient.

\section{Oral maintenance therapy}

The commonly considered agents are briefly discussed below. The ideal agent for use during pregnancy should have the following characteristics: ease of administration, rapid onset of action, controlled lowering of blood pressure, a sustained duration of action, maintenance of placental blood flow and lack of serious side effects for the mother and fetus. Classes of agents may be combined when maximum doses are inadequate to achieve the desired blood pressure goals. An increasing body of evidence suggests that maternal hypertensive diseases during pregnancy are associated with adverse neurocognitive outcomes in children. ${ }^{(4)}$ It is therefore difficult to separate the effects of the disease and the therapy but evidence suggests that methyldopa, labetalol and nifedipine are the safer agents. ${ }^{(41,42)}$

Methyldopa (Class B), which acts as a false neurotransmitter in the central nervous system is widely regarded as the first choice agent during pregnancy. It has no effect on the cardiac output. Importantly, it has been prospectively evaluated in randomised clinical trials and has been shown to be safe for the mother, neonate and infant. Unfortunately methyldopa has only mild potency with a low oral bioavailability and a slow onset of action. Sedation and depression are both recognised side effects when high doses are used. It should therefore be avoided in the postpartum period. The typical dosage starts with $250 \mathrm{mg} 8$-hourly, increased as necessary to a maximum of $750 \mathrm{mg} 8$-hourly. When a second agent is needed to control maternal blood pressure the patient should be referred to an institution with specialist care.

Nifedipine (Class C), is a widely used calcium channel blocking agent. The use of nifedipine in pregnancy has been fairly well investigated. A number of randomised controlled trials have shown nifedipine to be an effective anti-hypertensive agent. ${ }^{(43-45)}$ It has been used for acute and maintenance therapy, moderate and severe degrees of hypertension and for chronic as well as pregnancy induced conditions. A useful attribute of nifedipine is that the degree of reduction in blood pressure it produces appears to be directly proportional to the pre-treatment pressure. ${ }^{(46)}$ It should be noted that with regards to uteroplacental perfusion, the normotensive situation differs substantially from pre-eclampsia. In normal pregnancies the uterine spiral arteries are converted to uteroplacental arteries by trophoblast invasion. Thus their muscular components are lost and a low-resistance/high-flow system is formed. In pre-eclampsia this fails to occur completely and uteroplacental flow is reduced. So, while calcium channel blocking agents have little direct effect on the normal uteroplacental bed, they may be able to exert a direct vasodilatory effect in pre-eclampsia, because of the partial preservation of the muscular component of the arteries. A study reporting on in-utero exposure to nifedipine and the development of children up to the age of 18 months shows the agent to be safe. ${ }^{(42)}$

Other reported benefits of calcium channel blockers include their rapid onset of action after oral administration, ${ }^{(40,47)}$ inhibition of platelet aggregation, ${ }^{(15)}$ decrease in erythrocyte 
aggregation, ${ }^{(48)}$ improved renal function ${ }^{(49)}$ and increased urinary excretion of prostacyclin metabolites. ${ }^{(50)}$ Despite causing vasodilatation, these agents seldom cause a problematic tachycardia. Transient facial flushing, headaches and warm sweaty extremities seem to be the most common side effects. The typical dosage starts with $30 \mathrm{mg}$ (slow release formulation) daily or 12-hourly as necessary, to a maximum of 120mg daily. ${ }^{(51)}$ The combination of a calcium channel blocker and $\mathrm{MgSO} 4$ has been shown to be safe. ${ }^{(52)}$

$\beta$-adrenergic blocking agents e.g. atenolol (Class D). The mechanism of action involves a reduction in cardiac output, a central adrenergic effect and a blockage of renin secretion. These agents may cause fetal intra-uterine growth restriction (IUGR) and neonatal hypoglycaemia, but may be used in the postpartum period. Labetalol (Class $C$ ) is a unique adrenoceptor antagonist as it has both $\alpha-1$ and non-selective $\beta$-blocking effects. It lowers blood pressure without compromising the maternal cardiovascular system by producing peripheral vasodilatation. $\beta$-blockers should be avoided in patients with asthma and congestive heart failure. The dose of oral labetalol begins at $200 \mathrm{mg}$ 12-hourly, increasing to a maximum of I $200 \mathrm{mg}$ constituted in 2-3 divided doses.

Hydralazine (Class C), acts directly on the vascular wall producing vasodilatation. When administered orally the effect is weak.

Diuretics e.g. hydrochlorothiazide (Class B). The use of these agents during pregnancy is controversial, largely due to the volume depletion associated with pre-eclampsia. Their use should be limited to women with early, severe chronic hypertension.

Angiotensin converting enzyme (ACE) inhibitors, angiotensin receptor blockers and direct renin inhibitors (all Class D), are contra-indicated in pregnancy as they have been linked to complications throughout pregnancy including congenital anomalies, IUGR, oligohydramnios and perinatal renal failure. ${ }^{(20)}$ Enalapril and captopril may be used with safety during lactation.

With oral maintenance therapy the majority of patients are controlled on methyldopa, and/or nifedipine. In problematic cases labetalol is useful.

\section{CONCLUSION}

There is no doubt that clinicians managing pregnant patients must have a thorough understanding of the management of hypertension. The choice of agents will vary according to the clinical situation but each clinical decision must be underpinned by knowledge of the agent to be used as well as the risks and benefits of such therapy to both the mother and the fetus or newborn infant.

\section{Conflict of interest: none declared.}

\section{REFERENCES}

I. Vest AR, Cho LS. Hypertension in pregnancy. Cardiol Clin 20 I 2;30:407-423.

2. Nelson-Piercy C. Handbook of obstetric medicine. London: Informa Healthcare 2006.

3. Abalos E, Cuesta C, Grosso AL, et al. Global and regional estimates of preeclampsia and eclampsia: a systematic review. Eur J Obstet Gynecol \& Reprod Biol 2013;170:1-7.

4. Pettit F, Brown MA. The management of pre-eclampsia: What we think we know. Eur J Obstet Gynecol Reprod Biol 20 I 2; 160:6-12.

5. Centre for Maternal and Child Enquiries (CMACE). Saving Mothers' Lives: reviewing maternal deaths to make motherhood safer: 2006-08. The eighth report on confidential enquiries into maternal deaths in the United Kingdom. BJOG 201 I; I I8(Suppl. I): I-203.

6. Saving Mothers 2008-2010: Fifth report on the confidential enquiries into maternal deaths in South Africa. 2012.

7. Adu-Bonsaffoh K, Samuel OA, Binlinla G, et al. Maternal deaths attributable to hypertensive disorders in a tertiary hospital in Ghana. Int J Gynecol Obstet 2013;123:110-113.

8. Hall DR, Grové D, Carstens E. Early pre-eclampsia: what proportion of women qualify for expectant management and if not, why not? Eur J Obstet Gynecol Reprod Biol 2006; I28:169-174.

9. Panday M, Mantel GD, Moodley J. Audit of severe acute morbidity in hypertensive pregnancies in a developing country. J Obstet Gynaecol 2004; 24: 387-391.

10. Say L, Souza J, Pattinson R. Maternal near miss - towards a standard tool for monitoring quality of maternal health care. Best practice \& research clinical obstetrics and gynaecology 2009;23:287-296.

I I. Odendaal ES, Steyn DW, Odendaal HJ. Obstetric causes for delivery of very low birth weight babies at Tygerberg Hospital. Seventeenth conference on priorities in perinatal care in Southern Africa, Aldam. March 1998.

12. Taliep Q, Theron G, Steyn DW, et al. Total perinatally related losses at Tygerberg Hospital: A comparison between 1986, 1993 and 2006. S Afr Med J 20। 0; 100:250-253.

13. Leunen K, Hall DR, Odendaal HJ, et al. The profile and complications of women with placental abruption and intra-uterine death. J Trop Paed 2003;49:231-234.

14. Hall DR. Abruptio placentae and disseminated intravascular coagulopathy. Semin Perinatol 2009;33:189-195.

15. Rubin PC, Butters L, McCabe R. Nifedipine and platelets in pregnancy. Am J Hypertens 1988; I:175-177.

16. Macklin R. Enrolling pregnant women in biomedical research. Lancet 2010;375:632-633.

17. Jim B, Sharma S, Kebede T, et al. Hypertension in pregnancy. Cardiology in review 2010;18:178-189.

18. Deak TM, Moskovitz JB. Hypertension and pregnancy. Emerg Med Clin N Am 2012;30:903-917.

19. Steegers EAP, von Dadelszen P, Duvekot J), et al. Pre-eclampsia. Lancet 2010;376:631-644.

20. Brown MA, Lindheimer MD, de Swiet M, et al. Editorial from ISSHP. The classification and diagnosis of the hypertensive disorders of pregnancy: Statement from the International Society for the Study of Hypertension Pregnancy (ISSHP). Hypertens Preg 200 I;20:ix-xiv.

21. Martin JN Jr, Thigpen BD, Moore RC, et al. Stroke and severe preeclampsia and eclampsia: a paradigm shift focusing on systolic blood pressure. Obstet Gynecol 2005; 105(2):246-254.

22. Von Dadelszen P, Sawchuck D, McMaster R, et al. The active implementation of pregnancy hypertension guidelines in British Columbia. Obstet Gynecol 2010; 1 16:659-666.

23. Sibai BM. Caring for women with hypertension in pregnancy. JAMA 2007;298:1566-1568. 


\section{REFERENCES}

24. American College of Obstetricians and Gynecologists. Chronic hypertension in pregnancy. ACOG practice bulletin no. 125. Obstet Gynecol 2012; 1 19:396-407.

25. Abalos E, Duley L, Steyn DW, et al. Antihypertensive drug therapy for mild to moderate hypertension during pregnancy. Cochrane Database Syst Rev 2012;issue 10.

26. Odendaal HJ, Pattinson RC, Bam R, et al. Aggressive or expectant management for patients with severe preeclampsia between 28-34 weeks gestation: A randomised controlled trial. Obstet Gynecol 1990;76:1070- 1075.

27. Sibai BM, Mercer BM, Schiff E, et al. Aggressive versus expectant management of severe preeclampsia at 28-32 week's gestation: A randomised controlled trial. Am J Obstet Gynecol 1994; 171:818-822.

28. Hall DR, Odendaal HJ, Kirsten GF, et al. Expectant management of early onset, severe pre-eclampsia: perinatal outcome. BJOG 2000; 107: I 258-1264.

29. National Collaborating Centre for Women's and Children's Health. Hypertension in pregnancy. The management of hypertensive disorders during pregnancy. London: National Institute for Health and Clinical Excellence (NICE); 20 10. p46. (Clinical Guideline; no. 107).

30. Saade GR. Pregnancy as a window to future health. Obstet Gynecol 2009; | | 4:958-960.

31. Magnussen EB, Vatten LJ, Smith GD, et al. Hypertensive diseases in pregnancy and subsequently measured cardiovascular risk factors. Obstet Gynecol 2009; 1 14:961-970.

32. Sugelle M, Herdse F, Seiler M, et al. Cardiovascular risk markers in pregnancies complicated by diabetes mellitus or preeclampsia. Preg Hypertens 2012; 2:403-4I0.

33. Van Veen TR, Panerai RB, Haeri S, et al. Cerebral autoregulation in normal pregnancy and pre-eclampsia. Obstet Gynecol 2013; 122: 1064- 1069.

34. Duley L, Henderson-Smart DJ, Meher S. Drugs for treatment of very high blood pressure during pregnancy. Cochrane Database Sys Rev 2013; 7:CD001449.

35. Langenegger $E$, Dalla S, Petro G, et al. Invasive versus non-invasive monitoring of acute severe hypertension in women with pre-eclampsia. Preg Hypertens 2012;2:374-379.

36. Hall DR. Acute renal failure. In: Cronje HS, Cilliers JBF, Pretorius editors Clinical Obstetrics, Third edition. Van Schaik Publishers, Paarl 201 1:699-703.

37. Grossman E, Messerli FH, Grodzicki T, et al. Should a moratorium be placed on sublingual nifedipine capsules given for hypertensive emergencies and pseudo-emergencies? JAMA 1996;276:1328-1331.

38. Magee LA, Cham C, Waterman EJ, et al. Hydralazine for treatment of severe hypertension in pregnancy: Meta-analysis. BMJ 2003;327:955-960.

39. Moodley J. Hypertensive emergencies in underresourced countries. Curr Opin Obstet Gynecol 2008;20:91-95.

40. Shekhar S, Sharma C, Thakur S, et al. Oral nifedipine or intravenous labetalol for hypertensive emergency in pregnancy. A randomised controlled trial. Obstet Gynecol 2013;122:1057-1063.

41. Koren G. Systematic review of the effects of maternal hypertension in pregnancy and antihypertensive therapies on child neurocognitive development. Reprod Toxicol 2013;39: I-5.

42. Bortolus R, Ricci E, Chatenoud L, Parazzini F. Nifedipine administered in pregnancy: Effect on the development of children at 18 months. BJOG 2000; 107:792-794.

43. Hall DR, Odendaal HJ, Steyn DW, et al. Nifedipine or prazosin as a second agent to control early severe hypertension in pregnancy: A randomised controlled trial. BJOG 2000; 107:759-765

44. Martins-Costa S, Ramos JG, Barros E, et al. Randomised controlled trial of hydralazine versus nifedipine in pre-eclamptic women with acute hypertension. Clin Exp Hypertens (B) 1992; 1 1:25-44
45. Sibai BM, Barton JR, Akl S, et al. A randomised prospective comparison of nifedipine and bed rest versus bed rest alone in the management of preeclampsia remote from term. Am J Obstet Gynecol 1992;167:879-884.

46. Braunwald E. Mechanism of action of calcium-channel-blocking agents. N Engl J Med 1982;307:1618-1627.

47. Walters BN, Redman CW. Treatment of severe pregnancy-associated hypertension with the calcium antagonist nifedipine. BJOG 1984;91:330-336.

48. Tranquilli AL, Garzetti GG, De Tommaso G, et al. Nifedipine treatment in preeclampsia reverts the increased erythrocyte aggregation to normal. Am J Obstet Gynecol 1992; 167:942-945.

49. Ismail AA, Medhat I, Tawfic TA, et al. Evaluation of calcium-antagonist (nifedipine) in the treatment of pre-eclampsia. Int J Gynecol Obstet 1993; 40:39-43.

50. Manninen A, Metsa-Ketala T, Tuimala R, et al. Nifedipine increases urinary excretion of prostacyclin metabolite in hypertensive pregnancy. Pharmacol Toxicol 1991;69:60-63.

51. Sibai BM. Chronic hypertension in pregnancy. Obstet Gynecol 2002;100: 369-377.

52. Magee LA, Miremadi S, Li J, et al. Therapy with both magnesium sulphate and nifedipine does not increase the risk of serious magnesium-related maternal side effects in women with pre-eclampsia. Am J Obstet Gynecol 2005:193:153-163. 